\title{
Biochemische Serumparameter bei in Gefangenschaft gehaltenen Gazellen
}

\author{
S. Hammer' ${ }^{1}$ C. Hammer ${ }^{1}$, J. Schulz' ${ }^{1}$ U. Wernery², M. Clauss ${ }^{3}$ \\ ${ }^{1}$ Al Wabra Wildlife Preservation, Sheik Saoud Bin Mohd. Bin Ali Al-Thani, P0 Box 44069, Oatar (Director: Dr. Sven Hammer) \\ ${ }^{2}$ Central Veterinary Research Labor, Dubai, Vereinigte Arabische Emirate \\ ${ }^{3}$ Abteilung für Zoo-, exotische Heim- und Wildtiere, Vetsuisse Fakultät der Universität Zürich, Schweiz
}

Schlüsselwörter:

Gazellen - Wildwieder-

käuer-biochemische

Serumparameter - Spuren-

elemente - Ernährung -

Enzymanalytik - Referenzbereich - AST
Key words:

Gazelles - wild ruminant serum biochemistry - trace elements - nutrition enzyme analysis - reference range - AST

\section{Zusammenfassung:}

Ziel der Untersuchung war, Serumparameter von klinisch unauffälligen Gazellen der AI Wabra Wildlife Preservation (AWWP), Qatar, mit Referenzbereichen von Haus- und anderen Wildwiederkäuern zu vergleichen, um einerseits Rückschlüsse auf den Ernährungszustand der Tiere zu ermöglichen und andererseits Referenzbereiche für die untersuchten Arten zu etablieren. Material und Methoden: Von 250 klinisch gesunden Tieren der Arten Sömmeringgazelle (Gazella soemmerringii), Spekegazelle (Gazella spekei), Dorkasgazelle (Gazella dorcas), Saudigazelle (Gazella saudiya), Echtgazelle (Gazella gazella), Arabische Kropfgazelle (Gazella subgutturosa marica) und Chinkaragazelle (Gazella benetti) wurden biochemische Serumparameter und Mineralgehalte gemessen. Ergebnisse: Hinsichtlich des Ernährungszustandes war die Versorgung mit Spurenelementen (Selen, Kupfer, Zink, Eisen) in der AWWP gemäß den gemessenen Serumgehalten gewährleistet. Die Gehalte an Phosphor, Gesamtprotein und Albumin deuteten jedoch auf eine suboptimale Fütterung hin, die vermutlich mit der minderen Qualität des in der Region verfügbaren Raufutters zusammenhängt. Die Gehalte an Natrium, Kalium, Kalzium, Magnesium, Chlor, Triglyzeriden, Cholesterin, Kreatinin sowie die ALT- und $\gamma$-GT-Aktivität lagen - wie bei anderen Wildwiederkäuern auch im Referenzbereich für Hauswiederkäuer, der daher für die Beurteilung dieser Parameter bei Wiederkäuern generelle Gültigkeit besitzen dürfte. Die Gehalte an Glukose, Harnstoff-Stickstoff, Kreatinkinase sowie die AP-Aktivität hingegen scheinen bei Wildwiederkäuern generell erhöht zu sein. Während andere Wildwiederkäuer für AST mit Hauswiederkäuern vergleichbare Werte aufweisen, ist die Aktivität dieses Enzyms bei Gazellen in dieser und in anderen Studien durchweg erhöht. Schlussfolgerung und klinische Relevanz: Die in der Untersuchung festgestellten Besonderheiten müssen bei der Beurteilung von Blutanalysen beachtet werden.

\section{Summary:}

Objective: This study aimed at comparing serum parameters of clinically healthy gazelles of AI Wabra Wildlife Preservation (AWWP), Qatar, with reference ranges of domestic and other wild ruminants, in order to gain, on the one hand, insight into the nutritional status of the animals, and, on the other hand, to establish reference ranges for the investigated species. Material and methods: Serum biochemistry parameters and mineral levels were measured in 250 clinically healthy individuals of the species Soemmering's gazelle ( $G a-$ zella soemmerringii), Speke's gazelle (Gazella spekei), Dorcas gazelle (Gazella dorcas), Saudi gazelle (Gazella saudiya), Mountain gazelle (Gazella gazella), Arabian goitered gazelle (Gazella subgutturosa marica) and Chinkara Pakistani gazelle (Gazella benetti). Results: With respect to the nutritional status, the supplementation with trace elements (selenium, copper, zinc, iron) was adequate at AWWP, according to measured serum levels. In contrast, serum levels of phosphorus, total protein and albumin indicated a suboptimal feeding situation, most likely due to the low quality of the roughage available in the region. The levels of sodium, potassium, calcium, magnesium, choride, triglycerides, cholesterol, creatinine, ALT- as well as GGT avtivity were - as in other wild ruminants - within the reference range of domestic ruminants, which therefore should be applicable to ruminants in general. The contents of glucose, blood urea nitrogen, creatine kinase and ALP, in contrast, seem to be generally elevated in wild ruminants. While other wild ruminants display an AST activity comparable to those of domestic ruminants, gazelles of both this and other studies had elevated values of this enzyme. Conclusion and clinical relevance: These peculiarities need to be accounted for when interpreting blood values.

Serum biochemistry parameters in captive gazelle species 


\section{Einleitung}

Al Wabra Wildlife Preservation (AWWP) im Staat Qatar ist eine private Zuchteinrichtung für Wildtiere des arabischen und ostafrikanischen Raumes von Scheich Saoud Bin Mohd. Bin Ali Al-Thani. Der Bestand an Wildwiederkäuern beträgt ca. 1600 Tiere von 24 Spezies, darunter 1500 Gazellen (Genus Gazella) 20 unterschiedlicher Arten bzw. Unterarten. Da das natürliche Vorkommen dieser Tiere in Wüsten- und Steppenregionen liegt, ist die Haltung in Qatar klimatisch unproblematisch. Fünf der in AWWP gehaltenen Gazellenarten bzw. Unterarten (Sömmeringgazelle [Gazella soemmerringii], Spekegazelle [Gazella spekei], Dorkasgazelle [Gazella dorcas], Saudigazelle [Gazella saudiya], Echtgazelle [Gazella gazella]) gelten gemäß der Roten Liste der IUCN als ,schutzbedürftig“ (12).

Blutuntersuchungen von erkrankten Individuen gehören zur alltäglichen Praxis bei der tierärztlichen Betreuung dieses Bestandes. Die Interpretation der dabei erhobenen Befunde richtet sich zunächst nach den für Hauswiederkäuer bekannten Vergleichswerten, doch können diese nur als erste Anhaltspunkte für die Beurteilung der Ergebnisse betrachtet werden. Referenzwerte für die entsprechenden Spezies sind nur in geringem Umfang publiziertso für die Grantgazelle in freier Wildbahn in Kenia (Gazella gran$t i$; 4) sowie für die Sömmeringgazelle (17), Dorkasgazelle (1) und Arabische Kropfgazelle (Gazella subgutturosa marica, 23). Im Verzeichnis des International Species Inventory System (ISIS) finden sich zusätzlich Angaben zur Spekegazelle. Ein Vergleich mit diesen Werten ist für den klinischen Alltag vermeintlich sinnvoller als einer mit Standardwerten von Hauswiederkäuern. Im Idealfall jedoch sollten die Messwerte mit denen von klinisch unauffälligen Tieren derselben Haltung verglichen werden. Aus diesem Grund wurden in der AWWP während Fang- und Umsetzaktionen von 250 klinisch unauffälligen Gazellen Blutproben genommen und biochemisch untersucht. Diese Proben dienten zugleich zur Kontrolle des Erfolgs einer Futterumstellung: Die in der Region kultivierten Raufuttermittel, die in der AWWP eingesetzt werden, erwiesen sich nach einer Mineralstoffanalyse im Jahr 2001 als defizitär an Phosphor, Kalzium, Zink und Kupfer (im Vergleich zum Erhaltungsbedarf von Hauswiederkäuern). In Einzelfällen war in den Jahren zuvor Osteomalazie als vermeintliche Folge der suboptimalen Phosphorversorgung offensichtlich geworden. Anhand eines Mineralsupplements sollte daher ein Ausgleich dieser Mangelzustände erfolgen.

\section{Material und Methoden}

\section{Haltung und Fütterung der Tiere}

Die Blutproben stammten vorwiegend von adulten Tieren beiderlei Geschlechts (121 männliche und 129 weibliche). Die Gazellen werden teils in gemischt-geschlechtlichen Gruppen einer Spezies, aber auch in Junggesellengruppen mit anderen Spezies in eingezäunten Gehegen gehalten. Die Fütterung erfolgt einmal täglich. Dabei wird das Grundfutter ad libitum verabreicht. Es setzt sich zusammen aus Grasheu mäßiger Qualität sowie Weizenkleie und Gerste im Verhältnis 1:1 vermischt mit einem Vitamin-Mineral-Supplement (Blattin ${ }^{\circledR}$, Höveler Spezialfutterwerke $\mathrm{GmbH} \& \mathrm{Co} \mathrm{KG}$, Langenfeld). Täglich erhalten die Tiere eine begrenzte Menge frisch geschnittener Luzerne. Einmal pro Woche werden frische Laub- und Palmblätter angeboten, außerdem haben die Tiere freien Zugang zu Wasser und einem Salzleckstein.

\section{Probenentnahme}

Die Proben wurden in den Jahren 2001-2003 entnommen. Die Blutentnahme fand in Gruppen im Rahmen von Umsetzaktionen statt. Die hier berichteten Ergebnisse stammen dabei ausschließlich von klinisch unauffälligen Tieren. Die Blutentnahme erfolgte unter manueller Fixation nach Fangen mit Netzen bzw. unter Fixation in einer Transportkiste. Allen Tieren wurden sofort nach Fang die Augen mit einem Handtuch bedeckt, wodurch die Gazellen unmittelbar ruhig wurden und keine Abwehrreaktionen zeigten (10). Blut wurde stets aus der Vena jugularis mittels 18-Gauge-Kanülen und Vacutainern in 10-ml-Glasröhrchen ohne Antikoagulans entnommen. Nach der Gerinnung wurden die Proben zentrifugiert und das Serum in 2-ml-Plastikröhrchen überführt. Die Serumröhrchen lagerten bei $-18{ }^{\circ} \mathrm{C}$ bis zum gekühlten Transport nach Dubai. Von ursprünglich 260 genommenen Proben wurden 10 aufgrund offensichtlicher Hämolyse von der Untersuchung ausgeschlossen. Damit standen für die Untersuchung 250 Proben von ebenso vielen Tieren zur Verfügung.

\section{Durchgeführte Untersuchungen}

Die biochemische Untersuchung der Serumproben führte das Central Veterinary Research Labor in Dubai, Vereinigte Arabische Emirate, durch. Das Labor arbeitete mit dem Hitachi Analyzer 191/192, der täglich intern und alle zwei Wochen extern auf Messgenauigkeit geprüft wird. Die interne Prüfung erfolgte anhand von standardisierten Proben und die externe durch Vergleich mit ca. 300 anderen Labors (External Quality Assurance Services, Bio-Rad Laboratories, 9500 Jeronimo Road, Irvine, CA 92618, USA). Die gemessenen Parameter waren Harnstoff-Stickstoff, Kreatinin, Glukose, Gesamtprotein, Albumin, Cholesterin, Triglyzeride, weiterhin die Enzyme Aspartat-Aminotransferase (AST), Laktatdehydrogenase (LDH), $\gamma$-Glutamyl-Transferase $(\gamma$-GT), alkalische Phosphatase (AP), Alanin-Aminotransferase (ALT), Kreatinkinase (CK), Amylase, die Mineralstoffe Natrium, Kalium, Chlorid, Kalzium, Phosphat, Magnesium sowie die Spurenelemente Kupfer, Selen, Zink und Eisen.

\section{Auswertung}

Innerhalb einer Spezies wurden geschlechtsspezifische Unterschiede mittels t-Test bewertet $(\mathrm{p}=0,05)$.

Zum Vergleich dienten die Referenzbereiche für Hauswiederkäuer $(15,20)$ sowie der Bereich der Mittelwerte, die Peinado et al. (18) im Rahmen einer größeren vergleichenden Studie bei 10 Spezies im Zoo gehaltener Wildwiederkäuer ermittelten.

\section{Ergebnisse}

Die Ergebnisse der blutchemischen Untersuchung sind in denTabellen 1-3 dargestellt. Dabei werden jeweils Mittelwert, Standardabweichung, Minimum und Maximum für jede Spezies angegeben.

Die Werte von männlichen und weiblichen Tieren zeigten von wenigen Ausnahmen abgesehen keine systematischen Unterschiede. Höhere Werte bei weiblichen Tieren ergaben sich für Se- 
Biochemische Serumparameter bei in Gefangenschaft gehaltenen Gazellen

S. Hammer, C. Hammer, J. Schulz, U. Wernery, M. Clauss

Tab. 1 Mineralstoffgehalte im Serum von Gazellen in Al Wabra im Vergleich mit Referenzbereichen von Hauswiederkäuern und bei Zoowiederkäuern gemessenen Werten

\begin{tabular}{|c|c|c|c|c|c|c|c|c|c|c|}
\hline & $\begin{array}{l}\text { Kalzium } \\
(\mathrm{mmol} / \mathrm{l})\end{array}$ & $\begin{array}{l}\text { Phosphat } \\
\text { (mmol/l) }\end{array}$ & $\begin{array}{l}\text { Natrium } \\
(\mathrm{mmol} / \mathrm{l})\end{array}$ & $\begin{array}{l}\text { Kalium } \\
(\mathrm{mmol} / \mathrm{l})\end{array}$ & $\begin{array}{l}\text { Magnesium } \\
\text { (mmol/l) }\end{array}$ & $\begin{array}{l}\text { Chlorid } \\
(\mathrm{mmol} / \mathrm{l})\end{array}$ & $\begin{array}{l}\text { Kupfer } \\
(\mu \mathrm{g} / \mathrm{dl})\end{array}$ & $\begin{array}{l}\text { Selen } \\
(\mu \mathrm{g} / \mathrm{l})\end{array}$ & $\begin{array}{l}\text { Zink } \\
(\mu \mathrm{g} / \mathrm{dl}\end{array}$ & $\begin{array}{l}\text { Eisen } \\
(\mu \mathrm{mol} / \mathrm{l})\end{array}$ \\
\hline \multirow{3}{*}{$\begin{array}{l}\text { G. benetti } \\
\text { Chinkaragazelle } \\
(n=29)\end{array}$} & 2,27 & 2,18 & 153 & 4,56 & 1,06 & 112 & 110 & 123 & 127 & 42,6 \\
\hline & $\pm 0,19$ & $\pm 0,83$ & \pm 5 & $\pm 0,67$ & $\pm 0,24$ & \pm 4 & \pm 35 & \pm 26 & \pm 42 & $\pm 17,8$ \\
\hline & $(1,7-2,5)$ & $(0,8-4,4)$ & $\mid(142-162)$ & $(2,95-5,98)$ & $(0,4-1,5)$ & (104-119) & (39-173) & $\begin{array}{l}(70-172) \\
\end{array}$ & (81-251) & $(8-80)$ \\
\hline \multirow{3}{*}{$\begin{array}{l}\text { G. gazella } \\
\text { Echtgazelle } \\
(n=24)\end{array}$} & 2,30 & 1,88 & 153 & 4,79 & 1,23 & 111 & 91 & 130 & 121 & 34,7 \\
\hline & $\pm 0,24$ & $\pm 0,62$ & \pm 6 & $\pm 0,80$ & $\pm 0,17$ & \pm 5 & \pm 30 & \pm 30 & \pm 49 & $\pm 15,8$ \\
\hline & $(1,8-2,8)$ & $(0,7-3,2)$ & (145-170) & $(3,58-5,98)$ & $(0,9-1,6)$ & (104-123) & (49-182) & $\begin{array}{l}(70-195) \\
\end{array}$ & (58-280) & $(7-66)$ \\
\hline \multirow{3}{*}{$\begin{array}{l}\text { G. subgutt. marica } \\
\text { Arabische Kropfgazelle } \\
(\mathrm{n}=37)\end{array}$} & 1,94 & 1,45 & 155 & 4,65 & 1,18 & 114 & 86 & 181 & 206 & 55,2 \\
\hline & $\pm 0,69$ & $\pm 0,55$ & \pm 15 & $\pm 0,61$ & $\pm 0,43$ & \pm 11 & \pm 29 & \pm 38 & \pm 524 & $\pm 22,7$ \\
\hline & $(0,5-3,9)$ & $(0,5-3,3)$ & (137-230) & $(3,31-5,87)$ & $(0,5-2,8)$ & (94-161) & (24-169) & (102-252) & $(44-3300)$ & $\begin{array}{l}(5-96) \\
\end{array}$ \\
\hline \multirow{3}{*}{$\begin{array}{l}\text { G. saudiya } \\
\text { Saudigazelle } \\
(\mathrm{n}=44)\end{array}$} & 2,42 & 2,29 & 150 & 4,55 & 1,26 & 108 & 88 & 108 & 112 & 44,8 \\
\hline & $\pm 0,22$ & $\pm 0,72$ & \pm 5 & $\pm 0,65$ & $\pm 0,36$ & \pm 6 & \pm 30 & \pm 44 & \pm 41 & $\pm 17,1$ \\
\hline & $(2,0-2,9)$ & $(1,1-4,2)$ & (130-158) & $(3,29-6,30)$ & $(0,7-3,1)$ & (88-121) & (15-186) & (18-182) & (58-278) & (5-98) \\
\hline \multirow{3}{*}{$\begin{array}{l}\text { G. soemmeringii } \\
\text { Sömmeringgazelle } \\
(\mathrm{n}=38)\end{array}$} & 2,24 & 2,43 & \begin{tabular}{l|l}
152 \\
\end{tabular} & 4,30 & 1,23 & 110 & 99 & 179 & 301 & 55,3 \\
\hline & $\pm 0,35$ & $\pm 0,88$ & \pm 4 & $\pm 0,83$ & $\pm 0,23$ & \pm 4 & \pm 26 & \pm 37 & \pm 691 & $\pm 18,1$ \\
\hline & $(1,4-2,9)$ & $(1,1-5,1)$ & $(142-160)$ & $(3,03-5,91)$ & $(0,9-1,8)$ & $(104-120)$ & (43-182) & $(116-259)$ & $(81-3390)$ & $(25-103)$ \\
\hline \multirow{3}{*}{$\begin{array}{l}\text { G. spekei } \\
\text { Spekegazelle } \\
(\mathrm{n}=24)\end{array}$} & 2,09 & 2,28 & 153 & 3,86 & 1,14 & 108 & 86 & \begin{tabular}{l|l}
134 \\
\end{tabular} & \begin{tabular}{l|l}
240 \\
\end{tabular} & 40,7 \\
\hline & $\pm 0,49$ & $\pm 0,80$ & \pm 10 & $\pm 0,94$ & $\pm 0,34$ & \pm 8 & \pm 41 & \pm 36 & \pm 701 & $\pm 19,5$ \\
\hline & $(0,4-2,5)$ & $(0,7-3,9)$ & (124-169) & $(2,67-5,86)$ & $(0,3-1,8)$ & (85-119) & (22-174) & (93-245) & (29-3520) & (14-89) \\
\hline \multirow{3}{*}{$\begin{array}{l}\text { G. dorcas } \\
\text { Dorkasgazelle } \\
(\mathrm{n}=54)\end{array}$} & 2,36 & 1,62 & 151 & 4,24 & 1,11 & 109 & 80 & 149 & \begin{tabular}{l|l}
135 \\
\end{tabular} & 43,0 \\
\hline & $\pm 0,17$ & $\pm 0,60$ & \pm 3 & $\pm 0,70$ & $\pm 0,22$ & \pm 3 & \pm 34 & \pm 29 & \pm 76 & $\pm 14,2$ \\
\hline & $(2,0-2,7)$ & $(0,7-3,2)$ & (145-163) & $(2,74-6,23)$ & $(0,7-2,3)$ & (104-118) & (35-190) & $(46-202)$ & $(42-430)$ & (5-72) \\
\hline Rind $^{1}$ & $2,3-2,8$ & $1,6-2,3$ & 135-157 & $3,5-4,5$ & $0,8-1,2$ & 95-110 & $80-120$ & $80-300$ & $80-140$ & 13-44 \\
\hline Schaf' & $2,1-2,7$ & $1,3-1,9$ & $140-160$ & $3,5-4,5$ & $0,8-1,2$ & 100-106 & $70-200$ & $80-400$ & $80-120$ & \\
\hline Ziege $^{1}$ & $2,2-2,8$ & $1,4-2,3$ & & & & & $70-120$ & $80-200$ & $65-270$ & \\
\hline $\begin{array}{l}\text { Andere Wildwieder- } \\
\text { käuer }(18)^{2}\end{array}$ & $1,73-2,57$ & $2,71-4,36$ & $137-158$ & $3,9-5,3$ & $0,66-1,08$ & $86,4-101$ & & & & \\
\hline
\end{tabular}

len (bei G. spekei, G. dorcas, G. benetti, G. saudiya signifikant) und Albumin (bei G. dorcas, G. benetti, G. saudiya, G. soemmeringii signifikant). Männliche Tiere wiesen höhere Werte für Kupfer (bei G. benetti, G. saudiya, G. subgutt. marica signifikant) und Phosphat auf (bei G. dorcas, G. benetti, G. saudiya, G. subgutt. marica signifikant).

Für Phosphat variierten die Spezies-Mittelwerte zwischen 1,45 und 2,43 mmol/l (Referenzbereich Hauswiederkäuer [RBHW]: 1,3-2,3 mmol/l). Für Gesamtprotein und Albumin ergab sich dieser Bereich mit 58,0-65,5 g/l (RB-HW: 55-80 g/l) bzw. 26,4-32,7 g/l (RB-HW: 24-42 g/l). Mit einem Bereich der Spezies-Mittelwerte von 7,0-11,0 mmol// lagen die Glukosewerte der untersuchten Gazellen (stressbedingt, s. Diskussion) über dem Referenzbereich für Hauswiederkäuer (2,2-3,3 mmol/l). Glei- ches traf für Harnstoff-Stickstoff (6,5-10,6 mmol/1 vs. RB-HW von 3,2-8,2 mmol/1), AP (387-1054 U/1 vs. RB-HW von $<340$ U/1), CK (211-749 U/1 vs. RB-HW von < 100 U/l) und AST $(103-271 \mathrm{U} / 1$ vs. RB-HW von $<80 \mathrm{U} / \mathrm{l}) \mathrm{zu}$.

\section{Diskussion}

Die Bewertung der Ergebnisse erfolgt zunächst hinsichtlich des Ernährungszustandes und der Spurenelementversorung in der AWWP. Im Anschluss werden die Ergebnisse generell mit denen anderer Wildwiederkäuer und den Referenzbereichen von Hauswiederkäuern verglichen. 
Tab. 2 Biochemische Serumparameter von Gazellen in Al Wabra im Vergleich mit Referenzbereichen von Hauswiederkäuern und bei Zoowiederkäuern gemessenen Werten

\begin{tabular}{|c|c|c|c|c|c|c|c|}
\hline Spezies & $\begin{array}{l}\text { Triglyzeride } \\
\text { (mmol/l) }\end{array}$ & $\begin{array}{l}\text { Cholesterin } \\
\text { (mmol/l) }\end{array}$ & $\begin{array}{l}\text { Glukose } \\
\text { (mmol/l) }\end{array}$ & $\begin{array}{l}\text { Kreatinin } \\
(\mu \mathrm{mol} / \mathrm{l})\end{array}$ & $\begin{array}{l}\text { Harnstoff- } \\
\text { Stickstoff } \\
\text { (mmol/l) }\end{array}$ & $\begin{array}{l}\text { Gesamt- } \\
\text { protein } \\
(\mathrm{g} / \mathrm{l})\end{array}$ & $\begin{array}{l}\text { Albumin } \\
(\mathrm{g} / \mathrm{l})\end{array}$ \\
\hline \multirow{3}{*}{$\begin{array}{l}\text { G. benetti } \\
\text { Chinkaragazelle } \\
(\mathrm{n}=29)\end{array}$} & - & - & - & 86,4 & 7,7 & 63,7 & 30,6 \\
\hline & & & & $\pm 19,7$ & $\pm 1,9$ & $\pm 8,1$ & $\pm 3,7$ \\
\hline & & & & $\begin{array}{l}(52-127) \\
\end{array}$ & $(4-12)$ & (51-93) & (18-38) \\
\hline \multirow{3}{*}{$\begin{array}{l}\text { G. gazella } \\
\text { Echtgazelle } \\
(\mathrm{n}=24)\end{array}$} & 0,41 & 1,36 & 7,0 & 91,4 & 8,9 & 64,2 & 26,4 \\
\hline & $\pm 0,37$ & $\pm 0,43$ & $\pm 3,6$ & $\pm 28,8$ & $\pm 3,3$ & $\pm 8,8$ & $\pm 4,5$ \\
\hline & $(0,1-1,7)$ & $(0,5-2,2)$ & $(3,2-17,0)$ & $\begin{array}{l}\text { (39-143) } \\
\end{array}$ & $\begin{array}{l}(5-21) \\
\end{array}$ & $(50-84)$ & $(16-35)$ \\
\hline \multirow{3}{*}{$\begin{array}{l}\text { G. subgutt. marica } \\
\text { Arabische Kropfgazelle } \\
(\mathrm{n}=37)\end{array}$} & 0,53 & 1,21 & 11,0 & 126,5 & 9,6 & 58,0 & 32,7 \\
\hline & $\pm 0,67$ & $\pm 0,43$ & $\pm 4,5$ & $\pm 176,3$ & $\pm 14,6$ & $\pm 8,9$ & $\pm 6,9$ \\
\hline & $(0,1-3,2)$ & $(0,4-2,5)$ & $(1,2-25,3)$ & (60-1162) & (3-95) & $(46-93)$ & $(14-55)$ \\
\hline \multirow{3}{*}{$\begin{array}{l}\text { G. saudiya } \\
\text { Saudigazelle } \\
(\mathrm{n}=44)\end{array}$} & 0,60 & 1,71 & 8,0 & 112,4 & 10,3 & 58,6 & 29,6 \\
\hline & $\pm 0,49$ & $\pm 0,58$ & $\pm 2,6$ & $\pm 59,6$ & $\pm 9,1$ & $\pm 6,2$ & $\pm 2,7$ \\
\hline & $(0,2-2,7)$ & $(0,8-3,8)$ & $(3,3-17,2)$ & $(46-392)$ & (3-52) & (49-79) & $(21-34)$ \\
\hline \multirow{3}{*}{$\begin{array}{l}\text { G. soemmeringii } \\
\text { Sömmeringgazelle } \\
(\mathrm{n}=38)\end{array}$} & 0,48 & 1,48 & 9,4 & 118,4 & 8,6 & 63,9 & 28,8 \\
\hline & $\pm 0,44$ & $\pm 0,34$ & $\pm 2,5$ & $\pm 17,2$ & $\pm 2,0$ & $\pm 5,3$ & $\pm 2,5$ \\
\hline & $(0,1-2,5)$ & $(0,8-2,4)$ & $(4,5-16,7)$ & (97-168) & $(5-13)$ & (53-79) & (24-34) \\
\hline \multirow{3}{*}{$\begin{array}{l}\text { G. spekei } \\
\text { Spekegazelle } \\
(\mathrm{n}=24)\end{array}$} & 0,76 & 1,39 & 9,5 & 98,0 & 10,6 & 65,5 & 28,0 \\
\hline & $\pm 0,55$ & $\pm 0,47$ & $\pm 4,3$ & $\pm 32,3$ & $\pm 8,1$ & $\pm 10,0$ & $\pm 4,6$ \\
\hline & $(0,1-2,5)$ & $(0,6-2,2)$ & $(1,1-17,1)$ & $(48-174)$ & (5-45) & $(48-94)$ & (18-38) \\
\hline \multirow{3}{*}{$\begin{array}{l}\text { G. dorcas } \\
\text { Dorkasgazelle } \\
(\mathrm{n}=54)\end{array}$} & 0,43 & 1,31 & 8,5 & 89,1 & 6,5 & 58,5 & 29,8 \\
\hline & $\pm 0,53$ & $\pm 0,38$ & $\pm 3,3$ & $\pm 18,6$ & $\pm 1,6$ & $\pm 5,5$ & $\pm 2,6$ \\
\hline & $(0,1-4,0)$ & $(0,7-2,3)$ & $(4,5-21,9)$ & $(51-145)$ & (4-14) & (48-78) & $(24-36)$ \\
\hline Rind $^{1}$ & $0,17-0,51$ & $2-4,5$ & $2,2-3,3$ & 88-177 & $3,2-6,8$ & $60-80$ & $35-42$ \\
\hline Schaf ${ }^{1}$ & $0,06-0,34$ & $1,2-1,9$ & $2,2-3,3$ & 53-124 & $4,3-8,2$ & 55-75 & $24-30$ \\
\hline Ziege $^{1}$ & & $2-3,4$ & $2,2-3,1$ & 44-106 & $3,2-8,2$ & $65-75$ & $27-39$ \\
\hline Andere Wildwiederkäuer $(18)^{2}$ & $0,09-0,83$ & $1,05-3,17$ & $5,71-11,2$ & $132-238$ & $4,73-12,2$ & $63,8-74,5$ & $36,6-46,3$ \\
\hline
\end{tabular}

\section{Mineralversorgung und Ernährungszustand}

Der Status von Wildtieren hinsichtlich ihres Mineralhaushaltes wird in der Regel anhand der bei Haustieren bekannten Referenzbereiche beurteilt-sowohl bei Tieren aus freier Wildbahn (z. B. 6) als auch bei Tieren im Zoo (z. B. 2). Im Vergleich mit den Referenzbereichen von Hauswiederkäuern erscheinen die Gazellen in der AWWP gut versorgt mit Kupfer, Eisen, Selen und Zink. Gründe für den höheren Selenstatus der weiblichen Tiere können nicht angeführt werden. Hingegen entspricht der höhere Kupferstatus der männlichen Tiere vergleichbaren Beobachtungen bei Hausund anderen Wildwiederkäuern $(3,9,21)$. Hier ist davon auszugehen, dass gravide Weibchen vermehrt Kupfer an ihre Feten weitergeben.
Die Gehalte der Elemente Narium, Kalium, Chlorid, Magnesium und Kalzium im Serum werden vom Organismus homöostatisch geregelt und geben somit keinen Hinweis auf die Versorgung. Die Ähnlichkeit der bei Gazellen ermittelten Bereiche mit denen bei Hauswiederkäuern und anderen Zoowiederkäuern spricht dafür, dass diese Parameter speziesunabhängig eine hohe Konstanz aufweisen.

Zur Beurteilung des Ernährungszustandes frei lebender Wildwiederkäuer bzw. der Qualität ihres Habitats kann der Gehalt an Phosphat im Serum herangezogen werden $(7,8,16)$. Dabei ist zu betonen, dass ein solcher Parameter vor allem den Vergleich zwischen verschiedenen Populationen ermöglicht und der Wert für die Einzeltieruntersuchung fraglich ist. So erwies sich Phosphat im Zoo als ungeeigneter Indikator für einen schlechten Allgemeinzustand (14). Allerdings wäre diesen Überlegungen gemäß zu erwarten, dass Zoo- 
Biochemische Serumparameter bei in Gefangenschaft gehaltenen Gazellen

S. Hammer, C. Hammer, J. Schulz, U. Wernery, M. Clauss

Tab. 3 Enzymaktivitäten im Serum von Gazellen in Al Wabra im Vergleich mit Referenzbereichen von Hauswiederkäuern und bei Zoowiederkäuern gemessenen Werten

\begin{tabular}{|c|c|c|c|c|c|c|c|}
\hline Spezies & $\begin{array}{l}\text { CK } \\
\text { (U//) }\end{array}$ & $\begin{array}{l}\text { LDH } \\
\text { (U/I) }\end{array}$ & $\begin{array}{l}\text { AST } \\
\text { (U/I) }\end{array}$ & $\begin{array}{l}\text { ALT } \\
\text { (U/I) }\end{array}$ & $\begin{array}{l}\gamma-\mathbf{G T} \\
(\mathrm{U} / \mathrm{I})\end{array}$ & $\begin{array}{l}\text { AP } \\
\text { (U/I) }\end{array}$ & $\begin{array}{l}\text { Amylase } \\
\text { (U/I) }\end{array}$ \\
\hline \multirow{3}{*}{$\begin{array}{l}\text { G. benetti } \\
\text { Chinkaragazelle } \\
(\mathrm{n}=29)\end{array}$} & 488 & 633 & 271 & 39 & 22 & - & - \\
\hline & \pm 562 & \pm 204 & \pm 333 & \pm 50 & \pm 10 & & \\
\hline & $(60-2898)$ & (323-1048) & (48-1405) & $(2-260)$ & $(6-55)$ & & \\
\hline \multirow{3}{*}{$\begin{array}{l}\text { G. gazella } \\
\text { Echtgazelle } \\
(\mathrm{n}=24)\end{array}$} & 623 & 495 & 169 & 35 & 28 & 387 & 165 \\
\hline & \pm 1013 & \pm 147 & \pm 202 & \pm 35 & \pm 9 & \pm 342 & \pm 54 \\
\hline & (59-3903) & $(223-870)$ & (46-1090) & (4-192) & $(11-48)$ & (86-1582) & (93-292) \\
\hline \multirow{3}{*}{$\begin{array}{l}\text { G. subgutt. marica } \\
\text { Arabische Kropfgazelle } \\
(\mathrm{n}=37)\end{array}$} & 557 & 291 & 124 & 19 & 18 & 426 & 112 \\
\hline & \pm 1280 & \pm 114 & \pm 130 & \pm 17 & \pm 6 & \pm 389 & \pm 206 \\
\hline & (28-7651) & $(41-600)$ & (24-589) & (3-82) & $(9-35)$ & (42-2023) & $(41-1316)$ \\
\hline \multirow{3}{*}{$\begin{array}{l}\text { G. saudiya } \\
\text { Saudigazelle } \\
(\mathrm{n}=44)\end{array}$} & 503 & 592 & 215 & 50 & 21 & 1054 & 330 \\
\hline & \pm 738 & \pm 374 & \pm 385 & \pm 193 & \pm 23 & \pm 1202 & \pm 467 \\
\hline & $\begin{array}{l}(69-4112) \\
\end{array}$ & (294-2568) & $\begin{array}{l}\text { (54-2443) } \\
\end{array}$ & (2-1297) & (7-157) & (91-5554) & (92-2459) \\
\hline \multirow{3}{*}{$\begin{array}{l}\text { G. soemmeringii } \\
\text { Sömmeringgazelle } \\
(\mathrm{n}=38)\end{array}$} & 211 & 459 & 103 & 31 & 31 & 497 & 169 \\
\hline & \pm 161 & \pm 141 & \pm 53 & \pm 11 & \pm 14 & \pm 419 & \pm 56 \\
\hline & (39-715) & $(221-989)$ & $(30-302)$ & $(14-74)$ & $(17-64)$ & $(101-2029)$ & $(80-328)$ \\
\hline \multirow{3}{*}{$\begin{array}{l}\text { G. spekei } \\
\text { Spekegazelle } \\
(\mathrm{n}=24)\end{array}$} & 749 & 445 & 213 & 24 & 52 & 421 & 208 \\
\hline & \pm 1261 & \pm 163 & \pm 285 & \pm 18 & \pm 61 & \pm 304 & \pm 135 \\
\hline & (35-5095) & (208-791) & (34-1401) & $(7-68)$ & $(15-301)$ & $(35-1090)$ & (72-592) \\
\hline \multirow{3}{*}{$\begin{array}{l}\text { G. dorcas } \\
\text { Dorkasgazelle } \\
(\mathrm{n}=54)\end{array}$} & 392 & 500 & 210 & 39 & 15 & 544 & 227 \\
\hline & \pm 485 & \pm 145 & \pm 192 & \pm 45 & \pm 10 & \pm 491 & \pm 193 \\
\hline & $\begin{array}{l}(36-2427) \\
\end{array}$ & (253-917) & $\begin{array}{l}69-1225) \\
\end{array}$ & (16-296) & (4-60) & $(104-2157)$ & (113-1452) \\
\hline Rind $^{1}$ & $<100$ & - & $<80$ & $<50$ & $<50$ & $<200$ & 40-161 \\
\hline Schaf' ${ }^{1}$ & $<25$ & - & $<75$ & $<14$ & $<32$ & $<100$ & - \\
\hline Ziege $^{1}$ & $<65$ & - & $<65$ & - & $<23$ & $<340$ & - \\
\hline Andere Wildwiederkäuer (18) ${ }^{2}$ & $29-413$ & $310-635$ & $23,4-46,6$ & $10,7-29,1$ & $5,04-43,2$ & $64,7-583$ & - \\
\hline
\end{tabular}

tiere Werte im oberen Referenzbereich aufweisen und höhere als Tiere aus freier Wildbahn, da eine optimale Ernährung in Menschenobhut gewährleistet sein sollte. Dementsprechend fanden sich bei Zoo-Gazellen höhere Phosphatwerte als bei Tieren derselben Spezies in freier Wildbahn (1). Ebenso wiesen die Zoo-Wiederkäuer der Untersuchung von Peinado et al. (18) generell vergleichsweise hohe Phosphatgehalte im Serum auf (und das, obwohl diese Tiere chemisch immobilisiert waren, was zu einer Erniedrigung der Phosphatkonzentration im Serum führt [5]). Die Gazellen aus der AWWP hingegen zeigten teilweise Werte, die im unteren Referenzbereich von Hauswiederkäuern und deutlich unter denen der Zootiere von Peinado et al. (18) liegen. Dies deutet zwar auf eine adäquate Versorgung mit diesem Mineralstoff hin, aber auch auf eine generelle suboptimale Fütterung. Diese Sichtweise wird durch die Befunde bezüglich Gesamtprotein und Albumin unterstützt. Auch bei diesen Parame- tern wiesen die Gazellen aus der AWWP Gehalte im unteren Referenzbereich bzw. leicht darunter auf, während die Zoo-Wiederkäuer von Peinado et al. (18) deutlich höhere Werte erzielten (trotz chemischer Immobilisation, die die Konzentration an Gesamtprotein im Serum erniedrigt [19]). Diese Ergebnisse sprechen dafür, dass die Fütterung in AWWP optimiert werden könnte - insbesondere hinsichtlich der Qualität des Raufutters, das in dieser Region allerdings sehr schwierig zu beschaffen ist.

\section{Biochemische Parameter bei Wildwiederkäuern}

Von den bereits angesprochenen Parametern (Gesamtprotein, Albumin, Narium, Kalium, Kalzium, Phosphat, Magnesium, Chlo- 
rid, Selen, Kupfer, Zink und Eisen) abgesehen weisen auch andere Parameter eine offenbar hohe interspezifische Konstanz auf und sind daher zwischen dieser Studie, dem Referenzbereich für Hauswiederkäuer und der Studie von Peinado et al. (18) vergleichbar, nämlich $\gamma$-GT, Triglyzeride, Cholesterin, Kreatinin und ALT (Achtung: beim Wiederkäuer unspezifisch). Es ist daher davon auszugehen, dass diese Parameter auch bei weiteren Wildwiederkäuern ohne Bestimmung eines speziesspezifischen Referenzbereiches diagnostisch genutzt werden können.

Bei einigen anderen Parametern ergeben sich allerdings scheinbar systematische Unterschiede zwischen Haus- und Wildwiederkäuern, da sowohl die Daten dieser Studie als auch die von Peinado et al. (18) gleichgerichtet von den bei Hauswiederkäuern etablierten Referenzbereichen abweichen. So ist bekannt, dass der für Wildtiere mit einer Blutentnahme verbundene Stress (unabhängig von der Methode zur Immobilisation) generell zu höheren Glukosewerten führt - so wie bei den Gazellen in Al Wabra und den Zootieren von Peinado et al. (18) offensichtlich. Bush et al. (1) fanden zwar auch bei den generell erhöhten Glukosespiegeln im Serum, dass akut traumatisierte Tiere einen verringerten Wert aufwiesen, doch ist die praktische Relevanz dieser Beobachtung fraglich.

Weniger bekannt ist, dass auch die Harnstoff-Stickstoff-Konzentration bei Wildwiederkäuern deutlich über dem Referenzbereich für Hauswiederkäuer liegen kann. Als Ursache dafür könnte vermutet werden, dass sich der Referenzbereich bei Haustieren auf präprandiale Messungen bezieht, während der zeitliche Abstand zur letzten Futteraufnahme bei den Gazellen der vorliegenden Studie nicht kontrolliert wurde. Auch die von Peinado et al. (18) untersuchten Tiere wiesen jedoch durchweg hohe Werte auf, obwohl diese Autoren Tiere nur vor der allmorgendlichen Fütterung beprobten. Gründe für diese erhöhten Harnstoff-Stickstoff-Gehalte bei Wildwiederkäuern sind unseres Wissens nach nicht bekannt. Daher kann nur spekuliert werden, ob sich dieser Wert auch infolge einer Stresseinwirkung erhöht. Bei der Beurteilung von Individuen frei lebender Populationen kann HarnstoffStickstoff auch als Indikator für die Habitatqualität genutzt werden $(6,16)$, und innerhalb der Individuen einer Population lassen sich saisonale Veränderungen dieses Parameters mit der Vegetationsperiode demonstrieren (11). Allerdings lagen auch bei diesen Untersuchungen die gemessenen Gehalte deutlich über den Referenzbereichen für Hauswiederkäuer. Ähnliche hohe HarnstoffStickstoff-Werte wurden auch von anderen Autoren für Dorkasgazellen (1), Arabische Kropfgazellen (23), Sömmeringgazellen (17) sowie Oryxantilopen (22) beschrieben.

Die erhöhten CK-Werte könnten ebenfalls mit dem für Wildtiere bei der Probennahme verbundenen Stress in Verbindung stehen. Zumindest in der vorliegenden Studie kann ein Selenmangel als Ursache für die erhöhten CK-Werte ausgeschlossen werden. Auch bei den Zoowiederkäuern von Peinado et al. (18) und bei Oryxantilopen (22) waren erhöhte CK-Werte zu beobachten. Das Gleiche gilt für die AP, deren Aktivität bei den Gazellen von Al Wabra ebenso über dem Referenzbereich für Hauswiederkäuer lag wie bei den Zoowiederkäuern von Peinado et al. (18), bei Dor- kas- und Grantgazellen (1) und bei Sömmeringgazellen (17). Bush et al. (1) demonstrierten sogar, dass die AP bei Gazellen vermutlich eher bei auffallend niedrigen Werten diagnostisch einsetzbar ist. In ihrer Auswertung hatten Tiere mit Abszessen, nach chirurgischen Eingriffen bzw. mit unspezifisch gestörtem Allgemeinbefinden vergleichsweise erniedrigte AP-Werte. Auch hier ist die praktische Relevanz für die Einzeltierdiagnostik fraglich.

Als Besonderheit der vorliegenden Studie können die auffallend hohen AST-Werte betrachtet werden. Dieser Parameter lässt sich als Hinweis für eine übermäßige Belastung der Muskulatur im Zusammenhang mit der Immobilisation für die Blutentnahme - interpretieren. Allerdings weichen in diesem Fall die Ergebnisse der Untersuchung von Peinado et al. (18) von dem bei den Gazellen beobachteten Muster ab. Die Wildwiederkäuer von Peinado et al. (18) zeigten eine AST-Aktivität im Referenzbereich von Haussäugetieren. Allerdings finden sich in der Literatur Hinweise dafür, dass dieser Parameter bei Gazellen generell höhere Werte aufweist, so bei Arabischen Kropfgazellen (23), bei Sömmeringgazellen (17) oder bei Dorkasgazellen (1). Bei Oryxantilopen (22) oder bei Gabelböcken (6) hingegen wurden wiederum keine außerhalb des Haustier-Referenzbereichs liegenden Werte für dieses Enzym gemessen. Ein erhöhter AST-Wert könnte demnach typisch für die Gruppe der Gazellenartigen sein.

\section{Fazit für die Praxis}

Die Relevanz der Enzymdiagnostik bei Wildtieren wurde von Kitchen (13) relativiert. Dieser Autor zeigte, dass bei Serumenzymen die analytische Variation so groß ist, dass bei speziesübergreifenden Vergleichen potenzielle speziesspezifische Unterschiede nicht zuverlässig entdeckt werden können und deshalb speziesspezifische Besonderheiten nicht überbewertet werden sollen. Ein Vergleich mit Standard-Referenzwerten für Hauswiederkäuer sei daher gerechtfertigt. Unsere Studie belegt, dass diese Beobachtung bei vielen Parametern auch für Gazellen zutrifft. Kitchen (13) betont, dass es für den Zootierpraktiker wichtig ist, stets dasselbe Labor zu verwenden und für sich selbst Referenzwerte zu schaffen - am besten für individuelle Tiere. Sollen Parameter zur Diagnostik herangezogen werden, die entweder bei Wildwiederkäuern generell oder bei Gazellen im Besonderen systematisch von den Referenzbereichen für Hauswiederkäuer abweichen, ist eine solche Vorgehensweise unumgänglich. Bei der Bewertung der Ergebnisse muss bedacht werden, dass die gemessenen Werte für Glukose, Harnstoff-Stickstoff, Kreatinkinase und AP bei Wildwiederkäuern generell im Vergleich zu Werten von Hauswiederkäuern erhöht sind. Für Gazellen im Besonderen trifft dies auch für die Aktivität der AST zu. Wenn für ein zu untersuchendes Tier keine an diesem Individuum vor der Ausprägung klinischer Symptomatik ermittelten Referenzwerte zur Verfügung stehen, ist die Aussagekraft dieser Parameter für die Diagnostik als gering einzustufen. 


\section{Danksagung}

Die vorliegende Studie wurde erst möglich mit der Unterstützung der Mitarbeiter des Central Veterinary Laboratory in Dubai, namentlich: Fr. Sh. Korah, Fr. R. Marina, Fr. A. Annie, Fr. Y. Thyagarajan und Hr. Y. Abubaker. Für die Analysearbeiten sei herzlich gedankt. Ein besonderer Dank gilt den Mitarbeitern der Al Wabra Wildlife Preservation, namentlich: Fr. Tina Lang, Dr. Abdi Arif und Hr. Abid Taha, für die vorbereitenden Arbeiten und Versendung der Proben.

\section{Literatur}

1. Bush M, Smith EE, Custer RS. Hematology and serum chemistry values for captive Dorcas gazelles: variations with sex, age and health status. J Wildl Dis 1981; 17: 135-43.

2. Clauss M, Dierenfeld ES. Susceptibility of yak to copper deficiency. Vet Rec 1999; 145: 436-7.

3. Dierenfeld ES, Dolensek EP, McNamara TS, Doherty JG. Copper deficiency in captive blesbok antelope. J Zoo Anim Med 1988; 19: 126-31.

4. Drevemo S, Grootenhuis JG, Karstad L. Blood parameters in Wild ruminants in Kenya. J Wildl Dis 1974; 10: 327-34.

5. Drevemo S, Karstad L. The effect of Xylazine and Xylazine-Etorphine-Acepromacine combination on some clinical and haematological parameters in Impala and Eland. J Wildl Dis 1974; 10: 377-82.

6. Dunbar MR, Velarde R, Gregg MA, Bray M. Health evaluation of a pronghorn antelope population in Oregon. J Wildl Dis 1999; 35: 496-510.

7. Franzmann AW, LeResche RE. Alaskan moose blood studies with emphasis on condition evaluation. J Wildl Manage 1978; 42: 334-51.

8. Franzmann AW, Schwartz CC, Johnson DC. Monitoring status (condition, nutrition, health) of moose via blood. Swedish Wildl Res 1987; Suppl. 1 281-7.

9. Gooneratne SR, Christensen DA. A survey of maternal copper status and fetal tissue copper concentrations in Saskatchewan bovine. Can J Anim Sci 1986; 69: 141-50.

10. Hammer S, Hammer C. Das magische Handtuch, eine Therapiehilfe. Arbeitstagung der Zootierärzte im deutschsprachigen Raum 2001; 21: 77-9.

11. Houston DB. A note on the blood chemistry of the Shiras moose. J Mamm 1969; 50: 826.

12. IUCN 2003. Red List of Threatened Species. www.redlist.org, eingesehen im Dezember 2004
13. Kitchen $\mathrm{H}$. Blood values - normal, variation, abnormal and average values Proc AAZV 1979; $1-5$

14. Kock RA. Health and nutrition of the moose at Whipsnade Park. Verh Ber Erkr Zootiere 1985; 27: 101-6.

15. Kraft W, Dürr U, Hrsg. Klinische Labordiagnostik in der Tiermedizin. Stuttgart, New York: Schattauer 2005.

16. LeResche RE, Seal US, Karns PD, Franzmann AW. A review of blood chemistry of moose and other cervidae with emphasis on nutritional assessment. Nat Can 1974; 101: 263-90.

17. Olsen JH, Jones H. Hematologic and serum chemistry values for captive Soemmering's gazelle (Gazella soemmeringii). Immobilization consultation. J Zoo Anim Med 1984; 15: 5-7.

18. Peinado VI, Celdran JF, Palomeque J. Blood chemistry values in some wild ruminants in captivity. Comp Haematol Int 1999; 9: 175-81.

19. Presidente PJA, Lumdsen JH, Presnell KR, Rapley WA, McCraw BM. Combination of etorphine and xylazine in captive white-tailed deer: II. Effects on hematologic, serum biochemical and blood gas values. J Wildl Dis 1973; 9 342-7.

20. Puls R. Mineral Levels in Animal Health, 2nd ed. Clearbrook, Canada: Sherpa Int 1994

21. Rombach EP, Barboza PS, Blake JE. Costs of gestation in an Arctic ruminant: copper reserves in muskoxen. Comp Biochem Physiol 2003; 134C: $157-68$.

22. Vassart M, Greth A. Hematological and serum chemistry values for Arabian oryx (Oryx leucoryx). J Wildl Dis 1991; 27: 506-8.

23. Vassart M, Greth A, de la Farge F, Braun JP. Serum chemistry values for Arabian Sand gazelles (Gazella subgutturosa marica). J Wildl Dis 1994; 30: 426-8.

Dr. Marcus Clauss

Abteilung für Zoo-, exotische Heim- und Wildtiere

Vetsuisse Fakultät

der Universität Zürich

Winterthurerstrasse 260

CH-8057 Zürich

E-Mail:mclauss@vetclinics.unizh.ch 